\title{
Endosonographic characteristics of submucosal tumors (SMT). Approach and algorithm
}

\author{
M. J. Varas Lorenzo \\ Unit of Eco-Doppler and Ecoendoscopy. Centro Médico Teknon. \\ Unit of Ecoendoscopy. Centro Internacional de Medicina Avanzada (CIMA). Barcelona, Spain \\ AED Action Protocol for Gastrointestinal Ultrasonography. Coordinators: M. Gómez Rubio, MD and José Souto Ruzo, \\ $M D$.
}

Varas Lorenzo MJ. Endosonographic characteristics of submucosal tumors (SMT). Approach and algorithm. Rev Esp Enferm Dig 2004; 96: 215-218.

\section{INTRODUCTION}

Submucosal lesions (SMLs) of the upper gastrointestinal tract (1-4) represent a significant diagnostic and therapeutic challenge to both endoscopists and gastroenterologists. Such lesions may be assessed by using multifrequency transendoscopic miniprobes, or endoscopic ultrasonography (EUS), either radial or linear.

Fine-needle puncture-aspiration (FNA) of SMTs should be guided by only linear EUS.

\section{EUS YIELD}

Submucosal lesions or abnormalities:

1. Can EUS or endoscopic ultrasonography recognize submucosal lesions and differentiate between extrinsic compression (spleen, liver, etc.) and gastrointestinal submucosal tumors?

Yes, it can with a sensitivity greater than $90 \%(3,5)$. According to our group's cumulative experience (more than 140 cases), sensitivity was $94 \%$.

2. Can EUS recognize a lesion's exact origin, size, and ultrasonographic pattern?

Yes, EUS can establish whether a tumor is within the gastrointestinal wall or otherwise. Regarding submucosal

\section{Recibido: 29-12-03.}

Aceptado: 12-01-04.

Correspondencia: M. J. Varas Lorenzo. Unidad de Eco-Doppler y Ecoendoscopia. Centro Médico Teknon. Unidad de Ecoendoscopia. Centro Internacional de Medicina Avanzada (CIMA). Barcelona tumors (lipomas, myoid tumors), EUS can identify the layer a tumor originates in as well as its size, and is also a good technique for the investigation of gastroesophageal SMT endosonographic pattern (score of 9 at Monaco Consensus in 2000) (4). It also successfully differentiates liquid from solid lesions.

3. Can EUS differentiate between benign and malignant (stromal) SMTs?

It can be done with a sensitivity above $80 \%$ (6-8). EUS was assigned a score of only 6 at the Consensus Meeting held in 2000 (4).

Classic malignant characteristics include: size greater than $3 \mathrm{~cm}$, nodular shape, irregular borders, surface ulceration, heterogeneous pattern, and presence of anechoic foci indicating intratumor necrosis (6). According to the experience collected by us and other authors (7), benign SMTs are usually smaller than $4 \mathrm{~cm}$ in size. The likelihood of malignancy is $0-11 \%$ when none of the following signs are present: size greater than $4 \mathrm{~cm}$, irregular borders, echogenic foci greater than $3 \mathrm{~mm}$ in size, and cystic foci greater than $4 \mathrm{~mm}$ in size. Should more than two of these signs be present, the likelihood of malignancy rises to $80-100 \%$ (7). The finding of irregular borders, cystic shapes, or presence of adenopathies indicates malignancy with a positive predictive value (PPV) of $100 \%$ (8).

4. Which is the role of transendoscopic miniprobes (MPs) in assessing SMTs?

Two independent studies demonstrated that accord between EUS and MPs was very high. In our experience, the sensitivity of EUS and MP was 95\%, and accord was almost $100 \%$ (10). Therefore, we feel that MPs may be safely used in daily clinical practice to assess SMTs, as most of these lesions are usually benign (9), small in size (less than $2 \mathrm{~cm}$ ), and not in need of any treatment.

5. How are SMTs approached and managed? An algorithm? (Fig. 1).

Once an imaging technique (radiography, ultrasonography, computerized tomography, magnetic resonance 


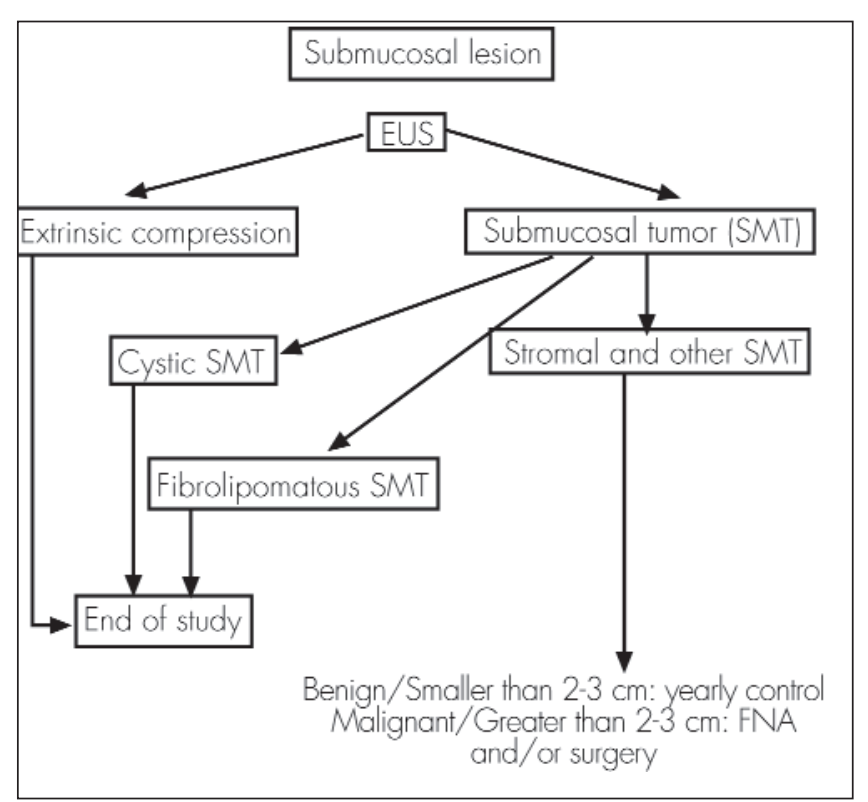

Fig. 1.- Every symptomatic SMT smaller than $2-3 \mathrm{~cm}$ arising from the second or third layer is eligible for endoscopic resection; those arising from the fourth layer are eligible for surgery, even of laparoscopic type. Carcinoid tumors smaller than $2 \mathrm{~cm}$ confined to the first three layers are eligible for endoscopic resection, whereas those greater in size growing into the fourth layer or in association with adenopathies must be managed using surgical resection. The treatment of choice for localized GISTs is complete surgical resection.

imaging, endoscopy) has detected the presence of a submucosal lesion, EUS must be unavoidably used to definitely confirm the presence of an SMT, attempt to characterize the lesion's histology, and orient regarding its bening or malignant nature. Studies will be terminated if the finding is a compression or a cystic or lipomatous SMT. Otherwise, surveillance with yearly follow-up or treatment by either endoscopic or surgical means is recommended, following a previous FNA with immunohistochemical testing [CD34, c-kit (CD117, Ki-67)] (11,12). Biopsy and FNA are of limited value, particularly for gastrointestinal stromal tumors (GISTs). A classification to predict aggression based on size, site, and mytotic index has been proposed for these tumors (13).

Symptomatic SMTs (bleeding, obstruction, etc.) smaller than $2-3 \mathrm{~cm}$ in size and originating in the second or third layer of the gut are eligible for endoscopic resection $(14,15)$ with low risk. Approximately, $75 \%$ of resected hypoechogenic SMTs are in fact GISTs, and at least 5\% of them are malignant (9).

\section{CONCLUSION}

EUS is essential in the assessment of SMLs. Most SMTs are benign and need not be treated. FNA may be used for cases not properly characterized by ultrasonography or doubtful. It is of limited value for GISTs. EUSprovided information may help decide on either endoscopic or surgical treatment.

\section{REFERENCES}

1. Yasuda K, Nakajima M, Yhosida S, Kiyota K, Kawai K. The diagnosis of submucosal tumors of the stomach by endoscopic ultrasonography. Gastrointest Endosc 1989; 35: 10-5.

2. Kawamoto K, Ueyama T, Iwashita I, et al. Colonic submucosal tumors: Comparison of Endoscopic US and target air-enema CT with barium enema study and colonoscopy. Radiology 1994; 192: 697702 .

3. Rösch T. Endosonografía en los tumores de la submucosa de vías gastrointestinales superiores: revisión de publicaciones. Clin Endoscopia Norteamérica 1995; 3: 593-7.

4. Lambert R, et al. EUS 2000. International Workshop on the clinical impact of endoscopic ultrasound in gastroenterology. Endoscopy 2000; 32: 549-84.

5. Varas MJ, Maluenda MD, Pou JM, Abad R, Turró J, Espinós JC. Valor de la ultrasonografía endoscópica en el estudio de los tumores submucosos del tracto digestivo. Gastroenterol y Hepatol 1998; 21: 121 4.

6. Yamada Y, Kida M, Sakaguchi T, et al. A study on myogenic tumor of upper gastrointestinal tract by endoscopic ultrasonography. Dig Endosc 1992; 4: 396-408.

7. Chak A, Canto MI, Rösch T, et al. Endosonographic differentiation of benign and malignant stromal cell tumors. Gastrointest Endosc 1997; 45: 468-73.

8. Palazzo L, Landi B, Séller C, et al. Endosonographic features predictive of benign and malignant gastrointestinal stromal cell tumors. Gut 2000; 46: 88-92.

9. Nickl N, Gress F, McClave S, et al. Hypoechoic intramural tumor study: final report. Gastrointest Endosc 2002; 55: AB98.

10. Varas MJ, Abad R, Maluenda MD, Pou JM. Ultrasonografía Endoscópica versus Minisonda en el estudio de los tumores submucosos del tracto digestivo. Rev Esp Eco Dig 2000; 2: 119-24.

11. Matsui M, Goto H, Niwa Y, et al. Preliminary results of fine needle aspiration biopsy histology in upper gastrointestinal submucosal tumours. Endoscopy 1998; 30: 750-5.

12. Ando N, Goto H, Niwa Y, Hirooka Y, Ohmiya N, et al. The diagnosis of GI stromal tumors with EUS-guided fine needle aspiration with immunohistochemiocal análisis. Gastrointest Endosc 2002; 55: 37 43.

13. Miettine M, El-Rifai W, Sobin LH, Lasota J. Evaluation og malignancy and prognosis of gastrointestinal stromal tumors: a review. Hum Pathol 2003; 33: 478-82.

14. Hyun JH, Jeen YT, Chun HJ, et al. Endoscopic resection of submucosal tumor of the esophagus: results in 62 patients. Endoscopy 1997; 29: $165-70$.

15. Hunt G, Smith P, Faigel D. Yield of tissue sampling for submucosal lesions evaluated by EUS. Gastrointest Endosc 2002; 56: S102. 


\title{
Características ecoendoscópicas de los tumores submucosos (TSM). Actitud a seguir y algoritmo
}

\author{
M. J. Varas Lorenzo \\ Unidad de Eco-Doppler y Ecoendoscopia. Centro Médico Teknon. \\ Unidad de Ecoendoscopia. Centro Internacional de Medicina Avanzada (CIMA). Barcelona \\ Protocolo de Actuación en Ecografía Digestiva de la AED. Coordinadores: Dr. M. Gómez Rubio y Dr. José Souto Ruzo
}

\section{INTRODUCCIÓN}

Las lesiones submucosas (LSM) del tracto digestivo (1-4) representan un importante reto diagnóstico y terapéutico para el endoscopista y para el gastroenterólogo. La evaluación de dichas lesiones se puede lograr con minisondas transendoscópicas multifrecuencia, ultrasonografía endoscópica (USE) radial o sectorial.

La punción con aguja fina (PAAF) de los TSM debe ser guiada exclusivamente mediante USE sectorial.

\section{RENDIMIENTO DE LA USE}

Lesiones o anormalidades submucosas:

1. ¿La USE o ecoendoscopia puede reconocer las lesiones submucosas y diferenciar entre compresiones extrínsecas (bazo, hígado, etc.) y tumores submucosos del tracto digestivo?

Sí, lo hace con una sensibilidad superior al $90 \%(3,5)$. En la experiencia acumulada por nuestro grupo (más de 140 casos), la sensibilidad fue del $94 \%$.

2. ¿Puede la USE reconocer el origen exacto de la lesión, su tamaño y su patrón ecográfico?

Sí, puede identificar si el tumor se encuentra en el exterior o en el interior de la pared gastrointestinal. En los tumores submucosos (lipomas, tumores mioides), puede identificar la capa donde se origina el tumor, su tamaño y representa una buena técnica para investigar el patrón ecoendoscópico de los TSM gastroesofágicos (puntuación de 9 en el Consenso de Mónaco del año 2000) (4). También diferencia muy bien las lesiones líquidas de las sólidas.

3. ¿Puede diferenciar la USE entre los TSM (del estroma) benignos y malignos?

Puede hacerlo con una sensibilidad superior al $80 \%$ (68). En la conferencia Consenso de Mónaco del año 2000 (4), se le asignó sólo una puntuación de 6.

Las características clásicas de malignidad son: tamaño superior a $3 \mathrm{~cm}$, forma nodular, borde irregular, ul- ceración superficial, patrón heterogéneo y presencia de focos anecoicos de necrosis intratumoral (6). En nuestra experiencia y en la de otros (7), los TSM benignos suelen ser menores de $4 \mathrm{~cm}$. Cuando no aparece ninguno de los siguientes signos: tamaño mayor de $4 \mathrm{~cm}$, borde irregular, focos ecogénicos mayores de $3 \mathrm{~mm}$ y focos quísticos mayores de $4 \mathrm{~mm}$, la probabilidad de malignidad es del 0-11\%. Si aparecen más de dos de estos signos, las probabilidades de malignidad ascienden al $80-100 \%$ (7). El hallazgo de un borde irregular, imágenes quísticas o la presencia de adenopatías asegura la malignidad del proceso con un valor predictivo positivo (VPP) del 100\% (8).

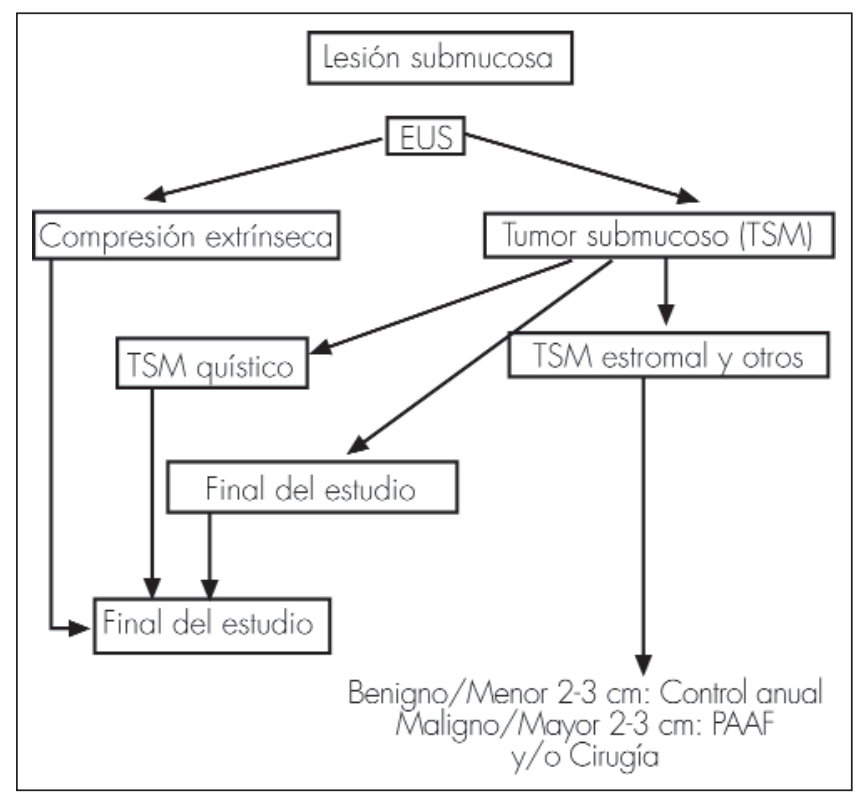

Fig. 1.- Cualquier TSM sintomático menor de $2-3 \mathrm{~cm}$, de la segunda o tercera capa, es candidato de resección endoscópica; los de la cuarta capa son candidatos de cirugía, incluso laparoscópica. Los tumores carcinoides menores de $2 \mathrm{~cm}$, confinados a las tres primeras capas son candidatos de resección endoscópica; mientras que los de mayor tamaño, que invadan la cuarta capa o estén asociados a adenopatías, deben ser tratados con resección quirúrgica. El tratamiento de elección de los GIST localizados es la resección quirúrgica completa. 
4. ¿Cuál es el papel de las minisondas transendoscópicas (MS) en la evaluación de los TSM?

Dos estudios independientes han demostrado que la concordancia entre la USE y las MS es muy alta. La sensibilidad de la USE y las MS en nuestra experiencia fue del $95 \%$ y la concordancia de casi el $100 \%$ (10). Por ello, creemos que las MS pueden ser utilizadas en la práctica clínica diaria para evaluar los TSM, ya que la mayoría de ellos suelen ser benignos (9), de pequeño tamaño (menores de $2 \mathrm{~cm}$ ) y no necesitan tratamiento.

5. ¿Cuál es el abordaje y manejo de los TMS? ¿Algoritmo? (Fig. 1).

Una vez que una técnica de imagen (radiografía, ecografía, tomografía computerizada, resonancia nuclear magnética, endoscopia) haya detectado la presencia de una lesión submucosa, debe realizarse ineludiblemente una USE para confirmar definitivamente la presencia del TSM, intentar caracterizar su tipo histológico y orientar su carácter benigno o maligno. Si se trata de una compresión o de un TSM quístico o fibrolipomatoso, se interrumpirán los estudios. En caso contrario, se recomienda bien su seguimiento mediante controles anuales o bien su tratamiento endoscópico o quirúrgico, tras haber realizado previamente una PAAF con estudio inmunohistoquí- mico [CD34, c-kit (CD117, Ki-67)] (11,12). El valor de la biopsia y la PAAF es limitado, principalmente en los tumores del estroma gastrointestinal (GISTs). Se ha propuesto una clasificación de predicción de la agresividad basada en el tamaño, localización e índice mitótico de estos tumores (13).

Los TSM sintomáticos (hemorragia, obstrucción, etc.), menores de $2-3 \mathrm{~cm}$ y originados en la segunda o tercera capa del tubo digestivo son candidatos de su resección endoscópica $(14,15)$ con escaso riesgo. Aproximadamente el $75 \%$ de los TSM hipoecogénicos resecados son GISTs y, a menos, el 5\% de ellos son malignos (9).

\section{CONCLUSIÓN}

La USE es esencial para la evaluación de las LSM. La mayoría de los TSM son benignos y no necesitan tratamiento. La PAAF puede utilizarse en aquellos casos que no hayan sido caracterizados adecuadamente mediante la ecografía o que sean dudosos. En los GISTs, su valor es limitado. La información obtenida con la USE puede ayudar a decidir el tratamiento endoscópico o quirúrgico. 